PROCEEDINGS OF THE

AMERICAN MATHEMATICAL SOCIETY

Volume 140, Number 11, November 2012, Pages 3749-3760

S 0002-9939(2012)11235-3

Article electronically published on March 12, 2012

\title{
ON THE RECURSION FORMULA FOR DOUBLE HURWITZ NUMBERS
}

\author{
SHENGMAO ZHU
}

(Communicated by Lev Borisov)

\begin{abstract}
In this paper, we will give a recursion formula for double Hurwitz numbers by the cut-join analysis. This recursion formula can be considered as a generalized version of the recursion formula for simple Hurwitz numbers derived by Mulase and Zhang. As a direct application, we get a polynomial identity for Goulden-Jackson-Vakil's conjectural intersection numbers and an explicit recursion formula for the computation of these intersection numbers with only $\psi$-classes.
\end{abstract}

\section{INTRODUCTION}

In this paper, we will follow the notation used in 7. Let $\mu=\left(\mu_{1}, \ldots, \mu_{m}\right)$ and $\nu=\left(\nu_{1}, \ldots, \nu_{n}\right)$ be two partitions of a positive integer $d$. The double Hurwitz number $H_{\mu, \nu}^{g}$ is the number of genus $g$ branched covers of $\mathbb{C P}^{1}$ with branching corresponding to $\mu$ and $\nu$ over 0 and $\infty$ respectively. By the Riemann-Hurwitz formula, such covers must have $r_{\mu, \nu}^{g}=(2 g-2+m+n)$ fixed simple branched points. As in [7], we take the extra condition that the points mapping to 0 and $\infty$ are labelled. Thus $H_{\mu, \nu}^{g}$ would be $|\operatorname{Aut}(\mu)| \cdot|\operatorname{Aut}(\nu)|$ bigger than the conventional definition [2, 8.

To understand the structure of double Hurwitz numbers is an interesting topic involving geometry, algebra and combinatorics.

We define $H_{\mu}^{g}:=H_{\mu,(1, ., 1)}^{g}$. Then $H_{\mu}^{g}$ is the simple Hurwitz number which has a rich structure.

The generating function of simple Hurwitz numbers $H_{\mu}^{g}$ is defined by

$$
H(\lambda, p)=\sum_{g \geq 0} \sum_{|\mu|>0} \frac{H_{\mu}^{g}}{|\operatorname{Aut}(\mu)|} p_{\mu} \frac{\lambda_{\mu}^{r_{\mu}^{g}}}{r_{\mu}^{g !}},
$$

which satisfies the following cut-join equation [6, 12, 19]:

$$
\frac{\partial H}{\partial \lambda}=\frac{1}{2}\left(\sum_{i, j \geq 1}(i+j) p_{i} p_{j} \frac{\partial H}{\partial p_{i+j}}+i j p_{i+j} \frac{\partial^{2} H}{\partial p_{i} \partial p_{j}}+i j p_{i+j} \frac{\partial H}{\partial p_{i}} \cdot \frac{\partial H}{\partial p_{j}}\right) .
$$

The celebrated Ekedahl-Lando-Shapiro-Vainshtein 2 formula relates Hurwitz numbers to the Hodge integrals on $\overline{\mathcal{M}}_{g, m}$ which is the Deligne-Mumford compactification of the moduli space of genus $g$ curves with $m$ marked points such that

Received by the editors November 30, 2010 and, in revised form, April 28, 2011.

2010 Mathematics Subject Classification. Primary 14H10; Secondary 05E05.

Key words and phrases. Hurwitz numbers, moduli space, cut-join, recursion.

(C)2012 American Mathematical Society Reverts to public domain 28 years from publication 
$2 g-2+m>0$

$$
H_{\mu}^{g}=r_{\mu}^{g} ! \prod_{i=1}^{m} \frac{\mu_{i}^{\mu_{i}}}{\mu_{i} !} \int_{\overline{\mathcal{M}}_{g, m}} \frac{1-\lambda_{1}+\lambda_{2}+\cdots+ \pm \lambda_{g}}{\left(1-\mu_{1} \psi_{1}\right) \cdots\left(1-\mu_{m} \psi_{m}\right)},
$$

where $\lambda_{k}$ and $\psi_{k}$ are the $\lambda$-class and $\psi$-class defined as follows: Denote by $\pi_{g, m+1}$ : $\overline{\mathcal{M}}_{g, m+1} \rightarrow \overline{\mathcal{M}}_{g, m}$ the natural projection. Let $\omega_{\pi_{g, m+1}}$ be the relative dualizing sheaf of $\pi_{g, m+1}$. The Hodge bundle on $\overline{\mathcal{M}}_{g, m}$ is defined by $\mathbb{E}=\left(\pi_{g, m+1}\right)_{*} \omega_{\pi_{g, m+1}}$. Then

$$
\lambda_{i}=c_{i}(\mathbb{E}) \in H^{2 i}\left(\overline{\mathcal{M}}_{g, m}, \mathbb{Q}\right) .
$$
Then

Let $\sigma: \overline{\mathcal{M}}_{g, m} \rightarrow \overline{\mathcal{M}}_{g, m+1}$ be the $i$-th section of $\pi_{g, m+1}$, and $\mathcal{L}_{i}=\sigma_{i}^{*}\left(\pi_{g, m+1}\right)$.

$$
\psi_{i}=c_{1}\left(\mathcal{L}_{i}\right) \in H^{2}\left(\overline{\mathcal{M}}_{g, m}, \mathbb{Q}\right) .
$$

The integrand on the right-hand side of formula (2) should be expanded formally, and then taking the terms of degree $3 g-3+n$ capped with the fundamental class $\overline{\mathcal{M}}_{g, m}$. We introduce the Witten notation of (linear) Hodge integrals:

$$
\left\langle\tau_{b_{1}} \tau_{b_{2}} \cdots \tau_{b_{m}} \lambda_{k}\right\rangle_{g}=\int_{\overline{\mathcal{M}}_{g, m}} \psi_{1}^{b_{1}} \cdots \psi_{m}^{b_{m}} \lambda_{k}
$$

which are zeros unless $\sum_{i=1}^{m} b_{i}+k=3 g-3+m$. We note that the original ELSV formula [2] includes a factor $|\operatorname{Aut}(\mu)|$, but here we consider the points over $\infty$ to be labelled.

The moduli spaces of curves have played an important role in diverse fields such as algebraic geometry, combinatorics, representation theory and mathematics physics. Many topological properties of the moduli spaces of curves are related to the computation of certain Hodge integrals [17, 3, 9, 4, 5, 13, 14, 18.

As stated above, the Hurwitz numbers relate to the linear Hodge integrals via the ELSV formula (2). Moreover, the generating function of Hurwitz numbers should satisfy the cut-join equation (1). Therefore, it is a natural idea to use the cutand-join restriction of Hurwitz numbers to get recursion formulas for linear Hodge integrals (3). Much progress has been achieved in this direction [16, 11, 8, 1, 10, 20, 21, 15. By cut-join analysis, due to Mulase and Zhang [15, formula (1) is equivalent to the following recursion formula for simple Hurwitz numbers:

(4) $(2 g-2+l(\mu)+|\mu|) H_{g}(\mu)$

$$
\begin{array}{r}
=\sum_{i<j}\left(\mu_{i}+\mu_{j}\right) H_{g}\left(\left(\mu(\hat{i}, \hat{j}), \mu_{i}+\mu_{j}\right)\right)+\frac{1}{2} \sum_{i=1}^{l} \sum_{\alpha+\beta=\mu_{i}} \alpha \cdot \beta\left(H_{g-1}((\mu(\hat{i}), \alpha, \beta))\right. \\
\left.+\sum_{\xi_{1} \amalg \xi_{2}=\mu(\hat{i})} \sum_{g_{1}+g_{2}=g} H_{g_{1}}\left(\xi_{1}, \alpha\right) H_{g_{2}}\left(\xi_{2}, \beta\right)\right),
\end{array}
$$

where $H_{g}(\mu)=\frac{H_{\mu}^{g}}{r_{\mu}^{g !}}$. Via some transformation of variables, they obtained a polynomial identity for linear Hodge integrals (Theorem 5.1 in [15]). As an application, they showed that this recursion implies the Witten-Kontsevich theorem when restricted to the top degree terms and the $\lambda_{g}$ formula when reduced to the lowest degree terms. 
Now, we come back to the double Hurwitz number $H_{\mu, \nu}^{g}$. We define the following generating function of $H_{\mu, \nu}^{g}$ :

$$
H=\sum_{g \geq 0} \sum_{d \geq 1} \sum_{\mu, \nu \vdash d} y^{d} z^{d} p_{\alpha} q_{\beta} u^{l(\beta)} \frac{H_{\mu, \nu}^{g}}{r_{\mu, \nu}^{g} !|A u t(\mu)| \cdot|A u t(\nu)|} .
$$

The following lemma is mentioned in 8$]$.

Lemma 1.1 (Cut-join equation).

$$
\begin{aligned}
& \left(\sum_{j \geq 1} p_{i} \frac{\partial}{\partial p_{i}}+u \frac{\partial}{\partial u}+2 y \frac{\partial}{\partial y}-2\right) H \\
& =\frac{1}{2}\left(\sum_{i, j \geq 1}(i+j) p_{i} p_{j} \frac{\partial H}{\partial p_{i+j}}+i j p_{i+j} y \frac{\partial^{2} H}{\partial p_{i} \partial p_{j}}+i j p_{i+j} \frac{\partial H}{\partial p_{i}} \cdot \frac{\partial H}{\partial p_{j}}\right)
\end{aligned}
$$

with initial conditions $\left[z^{i} p_{i} q_{i} u\right] H=\frac{1}{i}$ for $i \geq 1$.

With the same analysis used in [15], we obtain a recursion formula for $H_{\mu, \nu}^{g}$ which is equivalent to formula (5).

Theorem 1.2. The functions $H_{g}(\mu, \nu)$ defined by $H_{g}(\mu, \nu)=\frac{H_{\mu, \nu}^{g}}{r_{\mu, \nu}^{g} !}$ satisfy the following recursion formula:

(6)

$$
\begin{aligned}
& (2 g-2+l(\mu)+l(\nu)) H_{g}(\mu, \nu) \\
& =\sum_{i<j}\left(\mu_{i}+\mu_{j}\right) H_{g}\left(\left(\mu(\hat{i}, \hat{j}), \mu_{i}+\mu_{j}\right), \nu\right)+\frac{1}{2} \sum_{i=1}^{l} \sum_{\alpha+\beta=\mu_{i}} \alpha \cdot \beta\left(H_{g-1}((\mu(\hat{i}), \alpha, \beta), \nu)\right. \\
& \left.\quad+\sum_{\xi_{1} \amalg \xi_{2}=\mu(\hat{i})} \sum_{g_{1}+g_{2}=g} \sum_{\nu_{1} \amalg \nu_{2}=\nu} H_{g_{1}}\left(\left(\xi_{1}, \alpha\right), \nu_{1}\right) H_{g_{2}}\left(\left(\xi_{2}, \beta\right), \nu_{2}\right)\right),
\end{aligned}
$$

where the notation $\left(\mu(\hat{i}, \hat{j}), \mu_{i}+\mu_{j}\right)$ and $(\mu(\hat{i}), \alpha, \beta)$ will be introduced at the beginning of Section 2.

Remark 1.3. When $\nu$ is the partition of $d$ with profile $\nu=(1, \ldots, 1)$, then

$$
H_{g}(\mu,(1, \ldots, 1))=d ! H_{g}(\mu),
$$

where $H_{g}(\mu)$ is a quantity related to simple Hurwitz numbers defined in [15] (formula (3.5) in [15]).

Substituting (7) into (6), the recursion formula (6) is reduced to the recursion formula for simple Hurwitz numbers (4).

Example 1.4. When $g=0$ and $\nu=(d)$,

$$
(l(\mu)-1) H_{0}(\mu,(d))=\sum_{i<j}\left(\mu_{i}+\mu_{j}\right) H_{0}\left(\left(\mu(\hat{i}, \hat{j}), \mu_{i}+\mu_{j}\right),(d)\right)
$$

with the initial value $H_{0}((d),(d))=\frac{1}{d}$. Solving the above recursion (8), we get $H_{0}(\mu,(d))=d^{l(\mu)-2}$. Hence, $H_{(d), \mu}^{0}=(l(\mu)-1) d^{l(\mu)-2}$, which was calculated in [7]. 
When $\nu=(d)$, Goulden, Jackson and Vakil [7] conjectured that there existed a moduli space $\overline{P i c}_{g, n}$ and an ELSV-type formula for Hurwitz numbers $H_{\mu,(d)}^{g}$.

Conjecture 1.5. For each $g \geq 0, n \geq 1,(g, n) \neq(0,1),(0,2), \mu \vdash d$ and the length of $\mu, l(\mu)$ is $l$,

$$
H_{\mu,(d)}^{g}=r_{\mu,(d)}^{g} ! d \int_{\overline{P i c}_{g, l}} \frac{\Lambda_{0}-\Lambda_{2}+\cdots+ \pm \Lambda_{2 g}}{\left(1-\mu_{1} \psi_{1}\right) \cdots\left(1-\mu_{l} \psi_{l}\right)},
$$

where $\overline{\text { Pic }}_{g, l}$ is the conjectured moduli space with a fundamental class $\left[\overline{\text { Pic }}_{g, l}\right]$ of dimension $4 g-3+l . \psi_{i}$ and $\Lambda_{i}$ are two natural classes defined on $\overline{P i c}_{g, l}$.

We introduce the notation of linear GJV intersection numbers,

$$
\left\langle\left\langle\tau_{b_{1}} \tau_{b_{2}} \cdots \tau_{b_{l}} \Lambda_{k}\right\rangle\right\rangle_{g}=\int_{\overline{P i c}_{g, l}} \psi_{1}^{b_{1}} \cdots \psi_{l}^{b_{l}} \Lambda_{k}
$$

which are zeros unless $\sum_{i=1}^{l} b_{i}+k=4 g-3+l$. We refer the reader to the original paper 7 for a more precise description of the conjectured moduli space $\overline{P i c}_{g, l}$ and the GJV intersection numbers.

With the similar variable transformation technique used in [15, we obtain the following polynomial identity:

Theorem 1.6. The linear GJV intersection numbers satisfy the following polynomial identity:

$$
\begin{aligned}
& (2 g-1+l) \sum_{b_{L} \geq 0} \sum_{k=0}^{g}(-1)^{k}\left\langle\left\langle\tau_{b_{L}} \Lambda_{2 k}\right\rangle\right\rangle_{g} \psi_{b_{L}}\left(y_{L}\right) \\
& =\sum_{\substack{a \geq 0 \\
b_{L \backslash\{i, j\}} \geq 0}} \sum_{i<j} \sum_{k=0}^{g}(-1)^{k}\left\langle\left\langle\tau_{a} \tau_{b_{L \backslash\{i, j\}}} \Lambda_{2 k}\right\rangle\right\rangle_{g} \psi_{b_{L \backslash\{i, j\}}}\left(y_{L \backslash\{i, j\}}\right) \\
& \times\left[\psi_{a+1}\left(y_{i}\right) \frac{y_{i}\left(y_{j}-1\right)}{y_{i}-y_{j}}-\psi_{a+1}\left(y_{j}\right) \frac{y_{j}\left(y_{i}-1\right)}{y_{i}-y_{j}}\right] \\
& +\frac{1}{2} \sum_{i=1}^{l} \sum_{\substack{a_{1} \geq 0 \\
a_{2} \geq 0 \\
b_{L \backslash\{i\}} \geq 0}} \sum_{k=0}^{g}(-1)^{k}\left\langle\left\langle\tau_{a_{1}} \tau_{a_{2}} \tau_{b_{L \backslash\{i\}}} \Lambda_{2 k}\right\rangle\right\rangle_{g-1} \psi_{b_{L \backslash\{i\}}}\left(y_{L \backslash\{i\}}\right) \psi_{a_{1}+1}\left(y_{i}\right) \psi_{a_{2}+1}\left(y_{i}\right),
\end{aligned}
$$

where we have used the multiple index $\tau_{b_{L}}=\prod_{i=1}^{l} \tau_{b_{i}}$ and $\psi_{b_{L}}\left(y_{L}\right)=\prod_{i=1}^{l} \psi_{b_{i}}\left(y_{i}\right)$. $\psi_{b_{i}}\left(y_{i}\right)$ is a polynomial of $y_{i}$ defined by

$$
\psi_{b_{i}}\left(y_{i}\right)=\left(\left(y_{i}^{2}-y_{i}\right) \frac{d}{d y_{i}}\right)^{b_{i}}\left(y_{i}-1\right)
$$

for $b_{i} \geq 0$.

Restricting the above polynomial identity to the top degree terms, we obtain a recursion formula for the GJV's pure $\psi$-class intersection numbers. 
THE RECURSION FORMULA FOR DOUBLE HURWITZ NUMBERS

Theorem 1.7. We have the following recursion formula for intersection numbers $\left\langle\left\langle\tau_{b_{L}}\right\rangle\right\rangle_{g}$ :

$$
\begin{aligned}
& \left\langle\left\langle\tau_{b_{L}}\right\rangle\right\rangle_{g}=\frac{1}{2 g-1+l}\left[\sum_{i<j}\left\langle\left\langle\tau_{b_{i}+b_{j}-1} \tau_{b_{L \backslash\{i, j\}}}\right\rangle\right\rangle_{g} \frac{\left(b_{i}+b_{j}\right) !}{b_{i} ! b_{j} !}\right. \\
& \left.+\frac{1}{2} \sum_{i=1}^{l} \sum_{a_{1}+a_{2}=b_{i}-3}\left\langle\left\langle\tau_{a_{1}} \tau_{a_{2}} \tau_{b_{L \backslash\{i\}}}\right\rangle\right\rangle_{g-1} \frac{\left(a_{1}+1\right) !\left(a_{2}+1\right) !}{b_{i} !}\right] .
\end{aligned}
$$

Example 1.8. When $g=0$, the above recursion is

$$
\left\langle\left\langle\tau_{b_{L}}\right\rangle\right\rangle_{0}=\frac{1}{l-1} \sum_{i<j}\left\langle\left\langle\tau_{b_{i}+b_{j}-1} \tau_{b_{L \backslash\{i, j\}}}\right\rangle\right\rangle_{0} \frac{\left(b_{i}+b_{j}\right) !}{b_{i} ! b_{j} !}
$$

with the solution

$$
\left\langle\left\langle\tau_{b_{L}}\right\rangle\right\rangle_{0}=\left(\begin{array}{c}
l-3 \\
b_{1}, \ldots, b_{l}
\end{array}\right)
$$

This paper will be arranged as follows. After introducing the identities that will be used in the cut-join analysis, we obtain the recursion formula of Theorem 1.2 for double Hurwitz numbers in Section 2. Then, in Section 3, we show how to derive the polynomial identity for linear GJV intersection numbers (9) and the explicit formula for $\left\langle\left\langle\tau_{b_{L}}\right\rangle\right\rangle_{g}(10)$.

\section{ReCursion formula for DOUble HuRWitz numbers}

In this section, we present some combinatorial identities which will be used in the proof of our main result.

Let $\mu=\left(\mu_{1}, \mu_{2}, \ldots, \mu_{l}\right)=\left(1^{m_{1}(\mu)} 2^{m_{2}(\mu)} \ldots\right)$ be a partition of $d$ with length $l$. Then $|\operatorname{Aut}(\mu)|=\prod_{j \geq 1} m_{j}(\mu)$. We have the following formulas related to partition $\mu$.

i) We denote by $\left(\mu(\hat{i}, \hat{j}), \mu_{i}+\mu_{j}\right)$ the partition obtained from $\mu$ by erasing $\mu_{i}, \mu_{j}$ and adding $\mu_{i}+\mu_{j}$. Then if $\mu_{i}<\mu_{j}$,

$$
\left(\mu(\hat{i}, \hat{j}), \mu_{i}+\mu_{j}\right)=1^{m_{1}(\mu)} 2^{m_{2}(\mu)} \cdots \mu_{i}^{m_{\mu_{i}}(\mu)-1} \cdots \mu_{j}^{m_{\mu_{j}}(\mu)-1} \cdots\left(\mu_{i}+\mu_{j}\right)^{m_{\mu_{i}+\mu_{j}}+1} \cdots ;
$$

hence by definition,

$$
\begin{aligned}
\left|\operatorname{Aut}\left(\mu(\hat{i}, \hat{j}), \mu_{i}+\mu_{j}\right)\right| & =\left(m_{\mu_{i}}(\mu)-1\right)\left(m_{\mu_{j}}(\mu)-1\right)\left(m_{\mu_{i}+\mu_{j}}(\mu)+1\right) \prod_{k \neq i, j} m_{\mu_{k}}(\mu) \\
& =|\operatorname{Aut}(\mu)| \cdot \frac{m_{\mu_{i}+\mu_{j}}(\mu)+1}{m_{\mu_{i}}(\mu) m_{\mu_{j}}(\mu)}
\end{aligned}
$$

The analogous identity also holds for the case $\mu_{i}>\mu_{j}$.

When $\mu_{i}=\mu_{j}$,

$$
\begin{gathered}
\left(\mu(\hat{i}, \hat{j}), 2 \mu_{i}\right)=1^{m_{1}(\mu)} 2^{m_{2}(\mu)} \cdots \mu_{i}^{m_{\mu_{i}}(\mu)-2} \cdots\left(2 \mu_{i}\right)^{m_{2 \mu_{i}}(\mu)+1} \cdots \\
\left|\operatorname{Aut}\left(\mu(\hat{i}, \hat{j}), 2 \mu_{i}\right)\right|=|\operatorname{Aut}(\mu)| \cdot \frac{m_{2 \mu_{i}}(\mu)+1}{\left(m_{\mu_{i}}(\mu)-1\right)\left(m_{\mu_{i}}(\mu)\right)}
\end{gathered}
$$


With a similar analysis, we have

ii) Let $(\mu(\hat{i}), a, b)$ be a partition obtained by replacing $\mu_{i}$ with two elements $a, b$ such that $a+b=\mu_{i}$. Then we have

$$
|\operatorname{Aut}(\mu(\hat{i}), a, b)|= \begin{cases}|\operatorname{Aut}(\mu)| \cdot \frac{\left(m_{a}(\mu)+1\right)\left(m_{b}(\mu)+1\right)}{m_{a}(\mu)}, & a \neq b \\ |\operatorname{Aut}(\mu)| \cdot \frac{\left(m_{a}(\mu)+1\right)\left(m_{a}(\mu)+2\right)}{m_{2 a}(\mu)}, & a=b\end{cases}
$$

iii) If $\nu_{1}$ and $\nu_{2}$ are two partitions such that their join is $\mu$, i.e. $\nu_{1} \amalg \nu_{2}=\mu$, then

$$
\left|\operatorname{Aut}\left(\nu_{1}\right)\right| \cdot\left|\operatorname{Aut}\left(\nu_{2}\right)\right|=|\operatorname{Aut}(\mu)| \cdot \frac{1}{\prod_{j \geq 1}\left(\begin{array}{c}
m_{j}(\mu) \\
m_{j}\left(\nu_{1}\right)
\end{array}\right)} .
$$

iv) If $\nu_{1} \amalg \nu_{2}=\mu(\hat{i})$, and $a+b=\mu_{i}$, then

$\left|\operatorname{Aut}\left(\nu_{1}, a\right)\right| \cdot\left|\operatorname{Aut}\left(\nu_{2}, b\right)\right|=\left\{\begin{array}{l}|\operatorname{Aut}(\mu)| \cdot \frac{\left(m_{a}(\mu)+1\right)\left(m_{b}(\mu)+1\right)}{m_{a+b}(\mu)} \cdot \frac{1}{\prod_{j \geq 1}\left(\begin{array}{c}m_{j}(\mu(\hat{i}), a, b) \\ m_{j}\left(\nu_{1}, a\right)\end{array}\right)}, \quad a \neq b, \\ |\operatorname{Aut}(\mu)| \cdot \frac{\left(m_{a}(\mu)+1\right)\left(m_{a}(\mu)+2\right)}{m_{2 a}(\mu)} \cdot \frac{m^{\prime}}{\prod_{j \geq 1}\left(\begin{array}{c}m_{j}(\mu(\hat{\imath}), a, b) \\ m_{j}\left(\nu_{1}, a\right)\end{array}\right)}, \quad a=b .\end{array}\right.$

Substituting $H$ to the cut-join equation (5) and collecting the coefficients of $y^{g} z^{d} p_{\alpha} q_{\beta} u^{l(\beta)}$, we have

$$
\begin{gathered}
{\left[y^{g} z^{d} p_{\alpha} q_{\beta} u^{l(\beta)}\right] L H S=(2 g-2+l(\mu)+l(\nu)) \frac{H_{g}(\mu, \nu)}{|\operatorname{Aut}(\mu)| \cdot|\operatorname{Aut}(\nu)|},} \\
{\left[y^{g} z^{d} p_{\alpha} q_{\beta} u^{l(\beta)}\right]\left(\frac{1}{2} \sum_{i, j \geq 1}(i+j) p_{i} p_{j} \frac{\partial H}{\partial p_{i+j}}\right)} \\
=\frac{1}{2} \sum_{i<j}\left(\mu_{i}+\mu_{j}\right) \frac{H_{g}\left(\left(\mu(\hat{i}, \hat{j}), \mu_{i}+\mu_{j}\right), \nu\right)}{\left|\operatorname{Aut}\left(\mu(\hat{i}, \hat{j}), \mu_{i}+\mu_{j}\right)\right| \cdot|\operatorname{Aut}(\nu)|} \times \begin{cases}2 \cdot \frac{m_{\mu_{i}+\mu_{j}}(\mu)+1}{m_{\mu_{i}}(\mu) \cdot m_{\mu_{j}}(\mu)}, & \mu_{i} \neq \mu_{j}, \\
\frac{m_{2 \mu_{i}}(\mu)+1}{\left(\begin{array}{c}
m_{\mu_{2}(\mu)}(\mu) \\
2
\end{array}\right)}, & \mu_{i}=\mu_{j} .\end{cases}
\end{gathered}
$$

Also,

$$
\begin{aligned}
& {\left[y^{g} z^{d} p_{\alpha} q_{\beta} u^{l(\beta)}\right]\left(\frac{1}{2} \sum_{i, j \geq 1} i j p_{i+j} y \frac{\partial^{2} H}{\partial p_{i} p_{j}}\right)} \\
& =\frac{1}{2} \sum_{i=1}^{l} \sum_{\alpha+\beta=\mu_{i}} \alpha \cdot \beta \\
& \quad \times \frac{H_{g-1}((\mu(\hat{i}), \alpha, \beta), \nu)}{|\operatorname{Aut}(\mu(\hat{i}), \alpha, \beta)| \cdot|\operatorname{Aut}(\nu)|} \times \begin{cases}\frac{\left(m_{\alpha}(\mu)+1\right)\left(m_{\beta}(\mu)+1\right)}{m_{\mu_{i}}(\mu)}, & \alpha \neq \beta, \\
\frac{\left(m_{\alpha}(\mu)+1\right)\left(m_{\alpha}(\mu)+2\right)}{m_{\mu_{i}}(\mu)}, & \alpha=\beta .\end{cases}
\end{aligned}
$$


Moreover,

$$
\begin{aligned}
& {\left[y^{g} z^{d} p_{\alpha} q_{\beta} u^{l(\beta)}\right]\left(\frac{1}{2} \sum_{i, j \geq 1} i j p_{i+j} y \frac{\partial H}{\partial p_{i}} \frac{\partial H}{\partial p_{j}}\right)} \\
& =\frac{1}{2} \sum_{i=1}^{l} \sum_{\alpha+\beta=\mu_{i}} \alpha \cdot \beta \\
& \times \sum_{\xi_{1} \amalg \xi_{2}=\mu(\hat{i})} \sum_{g_{1}+g_{2}=g} \sum_{\nu_{1} \amalg \nu_{2}=\nu} \frac{H_{g_{1}}\left(\left(\xi_{1}, \alpha\right), \nu_{1}\right) H_{g_{2}}\left(\left(\xi_{2}, \beta\right), \nu_{2}\right)}{\left|\operatorname{Aut}\left(\xi_{1}, \alpha\right)\right| \cdot\left|\operatorname{Aut}\left(\xi_{2}, \beta\right)\right| \cdot\left|\operatorname{Aut}\left(\nu_{1}\right)\right| \cdot\left|\operatorname{Aut}\left(\nu_{2}\right)\right|} \\
& \cdot \frac{1}{\prod_{j \geq 1}\left(\begin{array}{c}
m_{j}(\nu) \\
m_{j}\left(\nu_{1}\right)
\end{array}\right)} \times \begin{cases}\frac{m_{\alpha}\left(\xi_{1}, \alpha\right) m_{\beta}\left(\xi_{2}, \beta\right)}{m_{\alpha+\beta}(\mu)} \cdot \frac{1}{\prod_{j \geq 1}\left(\begin{array}{c}
m_{j}(\mu(\hat{i})) \\
m_{j}\left(\xi_{1}\right)
\end{array}\right)}, \quad \alpha \neq \beta, \\
\frac{m_{\alpha}\left(\xi_{1}, \alpha\right) m_{\alpha}\left(\xi_{2}, \beta\right)}{m_{2 \alpha}(\mu)} \cdot \frac{1}{\prod_{j \geq 1}\left(\begin{array}{c}
m_{j}(\mu(\hat{\imath})) \\
m_{j}\left(\xi_{1}\right)
\end{array}\right)}, \quad \alpha=\beta .\end{cases}
\end{aligned}
$$

Combining the above analysis, we obtain Theorem 2.1.

\section{Polynomial ReCursion formula}

FOR GOULDEN-JACKSON-VAKIL'S INTERSECTION NUMBERS

Now, we focus on the special case for $\nu=(d)$. Then the formula $(6)$ can be written as

$$
\begin{aligned}
& (2 g-1+l) H_{g}(\mu,(d))=\sum_{i<j}\left(\mu_{i}+\mu_{j}\right) H_{g}\left(\left(\mu(\hat{i}, \hat{j}), \mu_{i}+\mu_{j}\right),(d)\right) \\
& +\frac{1}{2} \sum_{i=1}^{l} \sum_{\alpha+\beta=\mu_{i}} \alpha \cdot \beta H_{g-1}((\mu(\hat{i}), \alpha, \beta),(d))
\end{aligned}
$$

According to Conjecture 1.5, we have

$$
\begin{aligned}
H_{g}(\mu,(d)) & =\frac{H_{\mu,(d)}^{g}}{r_{\mu,(d)}^{g} !} \\
& =d \cdot \int_{\overline{P i c}_{g, l}} \frac{\Lambda_{0}-\Lambda_{2}+\cdots \pm \Lambda_{2 g}}{\left(1-\mu_{1} \psi_{1}\right) \cdots\left(1-\mu_{2} \psi_{2}\right)} \\
& =d \cdot \sum_{b_{i} \geq 0} \sum_{k=0}^{g}(-1)^{k}\left\langle\left\langle\tau_{b_{1}} \tau_{b_{2}} \cdots \tau_{b_{n}} \Lambda_{2 k}\right\rangle\right\rangle_{g} \mu_{1}^{b_{1}} \mu_{2}^{b_{2}} \cdots \mu_{n}^{b_{n}} .
\end{aligned}
$$

As $H_{\mu,(d)}$ is a symmetric function of $\mu_{1}, \ldots, \mu_{l}$, it is natural to extend its definition to $\mu \in \mathbb{N}^{l}$.

Let us introduce the generating function

$$
H_{g}\left(x_{1}, \ldots, x_{l}\right)=\sum_{\mu \in \mathbb{N}^{l}} H_{g}(\mu,(d)) \cdot \prod_{i=1}^{l} x_{i}^{\mu_{i}}
$$


Then

$$
\begin{aligned}
H_{g}\left(x_{1}, x_{2}, \ldots, x_{l}\right) & =\sum_{\mu \in \mathbb{N}^{l}} d \sum_{b_{L} \geq 0} \sum_{k=0}^{g}\left\langle\left\langle\tau_{b_{L}} \Lambda_{2 k}\right\rangle\right\rangle_{g} \mu_{L}^{b_{L}} x_{L}^{\mu_{L}} \\
& =d \cdot \sum_{b_{L} \geq 0} \sum_{k=0}^{g}(-1)^{k}\left\langle\left\langle\tau_{b_{L}} \Lambda_{2 k}\right\rangle\right\rangle_{g} \phi_{b_{L}}\left(x_{L}\right),
\end{aligned}
$$

where $\phi_{b_{L}}\left(x_{L}\right)=\prod_{i=1}^{l} \phi_{b_{i}}\left(x_{i}\right)$, and

$$
\phi_{b_{i}}\left(x_{i}\right)=\sum_{\mu_{i} \geq 1} \mu_{i}^{b_{i}} x_{i}^{\mu_{i}}=\left(x_{i} \frac{d}{d x_{i}}\right)^{b_{i}}\left(\sum_{\mu_{i} \geq 1} x_{i}^{\mu_{i}}\right)=\left(x_{i} \frac{d}{d x_{i}}\right)^{b_{i}}\left(\frac{1}{1-x_{i}}-1\right)
$$

when $b_{i} \geq 0$.

We now introduce the new variable $y_{i}=\frac{1}{1-x_{i}}$. It is easy to show that

$$
\left(y_{i}^{2}-y_{i}\right) \frac{d}{d y_{i}}=x_{i} \frac{d}{d x_{i}} .
$$

With the new variable $y_{i}$,

$$
\phi_{b_{i}}\left(x_{i}\right)=\left(\left(y_{i}^{2}-y_{i}\right) \frac{d}{d y_{i}}\right)^{b_{i}}\left(y_{i}-1\right) .
$$

We let

$$
\psi_{b_{i}}\left(y_{i}\right)=\left[\left(y_{i}^{2}-y_{i}\right) \frac{d}{d y_{i}}\right]^{b_{i}}\left(y_{i}-1\right),
$$

which is a polynomial of $y_{i}$.

Thereby,

$$
H_{g}\left(x_{1}, \ldots, x_{l}\right)=d \sum_{b_{L} \geq 0} \sum_{k=0}^{g}(-1)^{k}\left\langle\left\langle\tau_{b_{L}} \Lambda_{2 k}\right\rangle\right\rangle_{g} \psi_{b_{L}}\left(y_{L}\right) .
$$

Now, let us apply the above variable transformation procedure to formula (6). The left-hand side is

$$
\begin{aligned}
& \sum_{\mu \in \mathbb{N}^{l}}(2 g-1+l) H_{g}(\mu,(d)) x_{1}^{\mu_{1}} \cdots x_{l}^{\mu_{l}} \\
& =(2 g-1+l) \cdot d \cdot \sum_{b_{L} \geq 0} \sum_{k=0}^{g}(-1)^{k}\left\langle\left\langle\tau_{b_{L}} \Lambda_{2 k}\right\rangle\right\rangle_{g} \psi_{b_{L}}\left(y_{L}\right) .
\end{aligned}
$$


In order to calculate the first term on the right-hand side, we first need the following lemma:

\section{Lemma 3.1.}

$$
\sum_{\mu_{i}, \mu_{j} \geq 1}\left(\mu_{i}+\mu_{j}\right)^{a+1} x_{i}^{\mu_{i}} x_{j}^{\mu_{j}}=\psi_{a+1}\left(y_{i}\right) \frac{y_{i}\left(y_{j}-1\right)}{y_{i}-y_{j}}-\psi_{a+1}\left(y_{j}\right) \frac{y_{j}\left(y_{i}-1\right)}{y_{i}-y_{j}} .
$$

Proof. Prove by direct calculation,

$$
\begin{aligned}
& \sum_{\mu_{i}, \mu_{j} \geq 1}\left(\mu_{i}+\mu_{j}\right)^{a+1} x_{i}^{\mu_{i}} x_{j}^{\mu_{j}} \\
= & \sum_{\mu_{i}, \mu_{j} \geq 1}\left(\mu_{i}+\mu_{j}\right)^{a+1} x_{i}^{\mu_{i}+\mu_{j}}\left(\frac{x_{j}}{x_{i}}\right)^{\mu_{j}} \\
= & \sum_{\mu_{i}, \mu_{j} \geq 0}\left(\mu_{i}+\mu_{j}\right)^{a+1} x_{i}^{\mu_{i}+\mu_{j}}\left(\frac{x_{j}}{x_{i}}\right)^{\mu_{j}}-\sum_{\mu_{i} \geq 1} \mu_{i}^{a+1} x_{i}^{\mu_{i}}-\sum_{\mu_{j} \geq 1} \mu_{j}^{a+1} x_{j}^{\mu_{j}} \\
= & \sum_{\nu \geq 0} \sum_{\mu_{j}=0}^{\nu} \nu^{a+1} x_{i}^{\nu}\left(\frac{x_{j}}{x_{i}}\right)^{\mu_{j}}-\phi_{a+1}\left(x_{i}\right)-\phi_{a+1}\left(x_{j}\right) \\
= & \phi_{a+1}\left(x_{i}\right) \frac{x_{i}}{x_{i}-x_{j}}-\phi_{a+1}\left(x_{j}\right) \frac{x_{j}}{x_{i}-x_{j}}-\phi_{a+1}\left(x_{i}\right)-\phi_{a+1}\left(x_{j}\right) \\
= & \phi_{a+1}\left(x_{i}\right) \frac{x_{j}}{x_{i}-x_{j}}-\phi_{a+1}\left(x_{j}\right) \frac{x_{i}}{x_{i}-x_{j}} \\
= & \psi_{a+1}\left(y_{i}\right) \frac{y_{i}\left(y_{j}-1\right)}{y_{i}-y_{j}}-\psi_{a+1}\left(y_{j}\right) \frac{y_{j}\left(y_{i}-1\right)}{y_{i}-y_{j}} .
\end{aligned}
$$

Hence, by Lemma 3.1, we finish the calculation of the first term on the right-hand side,

$$
\begin{aligned}
& \sum_{\mu \in \mathbb{N}^{l}} \sum_{i<j}\left(\mu_{i}+\mu_{j}\right) H_{g}\left(\left(\mu(\hat{i}, \hat{j}), \mu_{i}+\mu_{j}\right),(d)\right) x_{1}^{\mu_{1}} x_{2}^{\mu_{2}} \cdots x_{l}^{\mu_{l}} \\
& =d \cdot \sum_{\substack{a \geq 0 \\
b_{L \backslash\{i, j\}} \geq 0}} \sum_{i<j} \sum_{k=0}^{g}(-1)^{k}\left\langle\left\langle\tau_{a} \tau_{b_{L \backslash\{i, j\}}} \Lambda_{2 k}\right\rangle\right\rangle_{g} \sum_{\mu \in \mathbb{N}^{l}} \mu_{L \backslash\{i, j\}}^{b_{L}} x_{L \backslash\{i, j\}}^{\mu_{L \backslash i, j\}}}\left(\mu_{i}+\mu_{j}\right)^{a+1} x_{i}^{\mu_{i}} x_{j}^{\mu_{j}} \\
& =d \cdot \sum_{\substack{a \geq 0 \\
b_{L \backslash\{i, j\}} \geq 0}} \sum_{i<j} \sum_{k=0}^{g}(-1)^{k}\left\langle\left\langle\tau_{a} \tau_{b_{L \backslash\{i, j\}}} \Lambda_{2 k}\right\rangle\right\rangle_{g} \psi_{b_{L \backslash\{i, j\}}}\left(y_{L \backslash\{i, j\}}\right) \\
& \quad \times\left[\psi_{a+1}\left(y_{i}\right) \frac{y_{i}\left(y_{j}-1\right)}{y_{i}-y_{j}}-\psi_{a+1}\left(y_{j}\right) \frac{y_{j}\left(y_{i}-1\right)}{y_{i}-y_{j}}\right] .
\end{aligned}
$$


Lastly, we need to calculate the second term on the right-hand side,

$$
\begin{aligned}
& \sum_{\mu \in \mathbb{N}^{l}} \frac{1}{2} \sum_{i=1}^{l} \sum_{\alpha+\beta=\mu_{i}} \alpha \cdot \beta H_{g-1}((\mu(\hat{i}), \alpha, \beta),(d)) x_{1}^{\mu_{1}} x_{2}^{\mu_{2}} \cdots x_{l}^{\mu_{l}} \\
& =\frac{1}{2} d \cdot \sum_{i=1}^{l} \sum_{\substack{a_{1} \geq 0 \\
a_{2} \geq 0 \\
b_{L} \backslash\{i\} \geq 0}} \sum_{\mu \in \mathbb{N}^{l}} \sum_{\alpha+\beta=\mu_{i}} \alpha \cdot \beta \\
& \times \sum_{k=0}^{g}(-1)^{k}\left\langle\left\langle\tau_{a_{1}} \tau_{a_{2}} \tau_{b_{L \backslash\{i\}}} \Lambda_{2 k}\right\rangle\right\rangle_{g-1} \alpha^{a_{1}} \beta^{a_{2}} \mu_{L \backslash\{i\}}^{b_{L \backslash\{i\}}} x_{L \backslash\{i\}}^{\mu_{L \backslash\{i\}}} x_{i}^{\alpha} x_{j}^{\beta} \\
& =\frac{1}{2} d \cdot \sum_{\substack{i=1 \\
a_{1} \geq 0 \\
a_{2} \geq 0 \\
b_{L} \backslash\{i\} \geq 0}}^{l} \sum_{k=0}^{g}(-1)^{k}\left\langle\left\langle\tau_{a_{1}} \tau_{a_{2}} \tau_{b_{L \backslash\{i\}}} \Lambda_{2 k}\right\rangle\right\rangle_{g-1} \psi_{b_{L \backslash\{i\}}}\left(y_{L \backslash\{i\}}\right) \psi_{a_{1}+1}\left(y_{i}\right) \psi_{a_{2}+1}\left(y_{i}\right) .
\end{aligned}
$$

Combining the formulas (12), (13) and (14) together, we obtain Theorem 1.6

As an application, a recursion formula for pure $\psi$-class intersection numbers $\left\langle\left\langle\tau_{b_{L}}\right\rangle\right\rangle_{g}$ will be derived from Theorem 1.6.

We need to take a close look at the polynomial $\psi_{b}(y)$ first. By definition,

$$
\psi_{0}(y)=y-1, \psi_{1}(y)=y^{2}-y, \psi_{2}(y)=2 y^{3}-3 y^{2}+y .
$$

More generally, $\psi_{b}(y)$ takes the following form:

$$
\psi_{b}(y)=\sum_{i=1}^{b+1} f(b, i) y^{i}
$$

By induction, we have the recursion relation for the coefficients $f(b, i)$,

$$
f(b, b+1)=f(b-1, b) b, f(b, i)=f(b-1, i-1)(i-1)-f(b, i) i
$$

for $b \geq 1,1 \geq i \leq b$. Thus, for a fixed $b$, all the coefficients $f(b, i)(1 \leq i \leq b)$ can be calculated through the above recursion. Particularly, we have $f(b, b+1)=b$ !.

From the above analysis, we know that $\psi_{b}(y)$ is a polynomial with degree $b+1$. So as a polynomial identity of variables $\left\{y_{i}\right\}$, formula (9) has highest degree $4 g-3+2 l$ when $\sum_{i=1}^{l} b_{i}=4 g-3+l$.

Let $F_{k}\left(g\left(y_{1}, \ldots, y_{l}\right)\right)$ be an operator by taking all the terms of the polynomial $g\left(y_{1}, \ldots, y_{n}\right) \in Q\left[y_{1}, \ldots, y_{n}\right]$ with degree $k$. 
With this notation,

$$
\begin{gathered}
F_{4 g-3+2 l}(\text { LHS of }(9))=(2 g-1+l) \sum_{b_{L} \geq 0}\left\langle\left\langle\tau_{b_{L}}\right\rangle\right\rangle_{g} b_{L} ! y_{L}^{b_{L}+1}, \\
F_{4 g-3+2 l}(\text { First term of RHS of }(9)) \\
=\sum_{\substack{a \geq 0 \\
b_{L} \backslash\{i, j\} \geq 0}} \sum_{i<j}\left\langle\left\langle\tau_{a} \tau_{b_{L \backslash\{i, j\}}}\right\rangle\right\rangle_{g} b_{L \backslash\{i, j\}} !(a+1) ! y_{L \backslash\{i, j\}}^{b_{L} \backslash\{i, j\}} \sum_{k=0}^{a+1} y_{i}^{a+2-k} y_{j}^{k+1}, \\
F_{4 g-3+2 l}(\text { Second term of RHS of }(9)) \\
=\frac{1}{2} \sum_{\substack{i=1 \\
\text { (16) } \\
b_{L} \backslash\{i\} \geq 0}} \sum_{\substack{a_{1} \geq 0 \\
a_{2} \geq 0}}^{g}(-1)^{k}\left\langle\left\langle\tau_{a_{1}} \tau_{a_{2}} \tau_{b_{L} \backslash\{i\}}\right\rangle\right\rangle_{g-1} b_{L \backslash\{i\}} !\left(a_{1}+1\right) !\left(a_{2}+1\right) ! y_{L \backslash\{i\}}^{b_{L \backslash i\}}+1} y_{i}^{a_{1}+a_{2}+4} .
\end{gathered}
$$

We denote by

$$
\left[y_{1}^{k_{1}} \cdots y_{n}^{k_{n}}\right] g\left(y_{1}, \ldots, y_{n}\right)
$$

the coefficient of $y_{1}^{k_{1}} \cdots y_{n}^{k_{n}}$ in the polynomial $g\left(y_{1}, \ldots, y_{n}\right) \in Q\left[y_{1}, \ldots, y_{n}\right]$.

Then,

$$
\begin{gathered}
{\left[y_{1}^{b_{1}+1}, \ldots, y_{l}^{b_{l}+1}\right](15)=(2 g-1+l)\left\langle\left\langle\tau_{b_{L}}\right\rangle\right\rangle_{g} b_{L} !} \\
{\left[y_{1}^{b_{1}+1}, \ldots, y_{l}^{b_{l}+1}\right](16)=\sum_{i<j}\left\langle\left\langle\tau_{b_{i}+b_{j}-1} \tau_{b_{L \backslash\{i, j\}}}\right\rangle\right\rangle_{g} b_{L \backslash\{i, j\}} !\left(b_{i}+b_{j}\right) !}
\end{gathered}
$$

$$
\left[y_{1}^{b_{1}+1}, \ldots, y_{l}^{b_{l}+1}\right](17)=\frac{1}{2} \sum_{i=1}^{l} \sum_{a_{1}+a_{2}=b_{i}-3}\left\langle\left\langle\tau_{a_{1}} \tau_{a_{2}} \tau_{b_{L} \backslash\{i\}}\right\rangle\right\rangle_{g-1} b_{L \backslash\{i\}} !\left(a_{1}+1\right) !\left(a_{2}+1\right) ! .
$$

Combining the formulas (18), (19) and (20) together, we obtain Theorem 1.7.

\section{ACKNOWLEDGEMENTS}

The author would like to thank Professor Kefeng Liu for bringing [7] to his attention. The author is grateful to the referee for valuable comments and suggestions, which greatly improved the presentation of the content.

\section{REFERENCES}

[1] L. Chen, Y. Li and K. Liu, Localization, Hurwitz numbers and the Witten conjecture, Asian J. Math. 12 (2008), 511-518. MR2481688(2009m:14084)

[2] T. Ekedahl, S. Lando, M. Shapiro, and A. Vainshtein, Hurwitz numbers and intersections on moduli spaces of curves, Invent. Math. 146 (2001), 297-327. MR.1864018(2002j:14034)

[3] C. Faber, A conjectural description of the tautological ring of the moduli space of curves. In Moduli of curves and abelian varieties, Aspects Math., E33, Vieweg, Braunschweig, Germany, 1999, 109-129. MR.1722541 (2000j:14044)

[4] C. Faber and R. Pandharipande, Hodge integrals, partition matrices, and the $\lambda_{g}$ conjecture, Ann. of Math. (2) 157 (2003), 97-124. MR.1954265 (2004b:14095)

[5] C. Faber and R. Pandharipande, Logarithmic series and Hodge integrals in the tautological ring (with an appendix by D. Zagier), Michigan Math. J. 48 (2000), 215-252. MR1786488 (2002e:14041)

[6] I.P. Goulden and D.M. Jackson, Transitive factorisations into transpositions and holomorphic mappings on the sphere, Proc. Amer. Math. Soc. 125 (1997), 51-60. MR.1396978 (97j:05007)

[7] I.P. Goulden, D.M. Jackson and R. Vakil, Towards the geometry of double Hurwitz numbers, Adv. Math. 198 (2005), 43-92. MR2183250(2006i:14023) 
[8] I.P. Goulden, D.M. Jackson and R. Vakil, A short proof of the $\lambda_{g}$-conjecture without GromovWitten theory: Hurwitz theory and the moduli of curves, J. Reine Angew. Math. 637 (2009), 175-191. MR2599085 (2011f:14087)

[9] E. Getzler and R. Pandharipande, Virasoro constraints and the Chern classes of the Hodge bundle, Nuclear Phys. B 530 (1998), 701-714. MR:1653492 (2000b:14073)

[10] M. Kazarian and S. Lando, An algebro-geometric proof of Witten's conjecture, J. Amer. Math. Soc. 20 (2007), 1079-1089. MR2328716 (2008d:14055)

[11] Y.S. Kim and K. Liu, A simple proof of Witten conjecture through localization, preprint, arXiv:math/0508384 [math.AG] (2005).

[12] A.M. Li, G. Zhao and Q. Zheng, The number of ramified coverings of a Riemann surface by Riemann surface, Commun. Math. Phys. 213 (2000), 685-696 . MR.1785434 (2001i:14078)

[13] K. Liu and $\mathrm{H}$. Xu, New results of intersection numbers on moduli spaces of curves, Proc. Natl. Acad. Sci. USA 104 (2007), 13896-13900. MR2348851 (2008f:14041)

[14] K. Liu and H. Xu, A proof of the Faber intersection number conjecture, J. Differential Geom. 83 (2009), 313-335. MR 2577471 (2011d:14051)

[15] M. Mulase and Naizhen Zhang, Polynomial recursion for linear Hodge integrals, Commun. Number Theory Phys. 4 (2010), 267-294. MR 2725053

[16] A. Okounkov and R. Pandharipande, Gromov-Witten theory, Hurwitz numbers, and matrix models, I, Proc. Symposia Pure Math. 80 (2009), 325-414. MR2483941 (2009k:14111)

[17] E. Witten, Two-dimensional gravity and intersection theory on moduli space, Surv. Differ. Geom. 1 (1991), 243-310. MR.1144529 (93e:32028)

[18] H. Xu, Hodge integrals on moduli spaces of curves, thesis submitted for the degree of Doctor of Philosophy of Zhejiang University, 2009.

[19] J. Zhou, Hodge integrals, Hurwitz numbers, and symmetric groups, arXiv:math/0308024.

[20] J. Zhou, On recursion relation for Hodge integrals from the cut-and-join equations, preprint, 2009.

[21] S. Zhu, Hodge integral with one $\lambda$-class, Sci. China Math. 55 (2012), doi:10.1007/S11425011-4313-7

Department of Mathematics and Center of Mathematical Sciences, Zhejiang UniverSity, Hangzhou, Zhejiang 310027, People's Republic of China

E-mail address: zhushengmao@gmail.com 